\title{
A cross-sectional study on the assessment of dermatology life quality index among the adolescents and young adults with acne vulgaris in south Indian population
}

\author{
Rathish T. Pillai ${ }^{1, *}$, Sanoof $\mathrm{MS}^{2}, \mathrm{Mysa}^{3}$ \\ ${ }^{1}$ Associate Professor, ${ }^{2}$ Junior Resident, ${ }^{3}$ Intern, Dept. of Dermatology, Azeezia Medical College, Kollam, Kerala, India \\ *Corresponding Author: \\ Email: sanoofms@gmail.com
}

\begin{abstract}
Acne vulgaris is one of the most common dermatoses involving chronic inflammation of hair follicles and their accompanying sebaceous gland. Depending up on the grade of the disease severity, it might have a psychological impact on any patient. There is a very few studies on the assessment of dermatology life quality index among patients with acne vulgaris in south India. Proper awareness and early intervention regarding the psychiatric sequel of acne can benefit the outcome of patients.

Materials and Methods: This is a hospital based prospective cross-sectional questionnaire study done on acne vulgaris patients attending the dermatology outpatient department in a tertiary care hospital in south Kerala. QoL was measured using both acne specific [Cardiff Acne Disability Index (CADI)] questionnaire and skin disease specific [Dermatology Life Quality Index (DLQI)] questionnaire.

Results: Among the patients assessed with DLQI questionnaire, $82.9 \%$ had impaired quality of life and $94.3 \%$ as assessed with the CADI. The mean CADI score was 5.8 and the mean DLQI score was 6.97. The impact of DLQI was more among adolescent age group than among young adults.

Conclusion: Acne vulgaris is a chronic inflammatory disease associated with increased likelihood for psychological burden in society especially among adolescent age group. It is vital for the dermatologist to incorporate quality of life index measurement when treating acne vulgaris patients to provide better emotional care and appropriate counselling.
\end{abstract}

Keyword: Acne, DLQI, QOL.

\section{Introduction}

Acne vulgaris is one of the most common dermatological disorder involving chronic inflammation of hair follicles and their accompanying sebaceous gland. Acne can present as noninflammatory lesions, inflammatory lesions, or a mixture of both, affecting mostly the face but also the back and chest. ${ }^{1}$ Acne can cause not only facial scarring but also leads to significant emotional distress and psychosocial implications on any patients regardless of the grade of disease severity. ${ }^{2}$ Low self-esteem due to affected personal appearance demoralizes the patients especially in the adolescent age group.

According to population based epidemiological studies, it is important to quantify social burden, distribution of the disease in the community and it's social and psychological impact, mainly in adolescent age group. Despite these facts, data based on community-based samples, including an actual dermatological examination of acne patients are lacking from India. ${ }^{3}$ Adolescent acne usually begins with the onset of puberty, when the gonads begin to produce and release more androgen hormone. Acne is not limited to adolescence. Twelve percent of women and $5 \%$ of men at aged 25 years have acne. By age 45 years, $5 \%$ of both men and women still have acne. ${ }^{4}$

Acne affects the quality of life. WHO defines Quality of Life as the "individual's perception of their position in the context of culture and value systems in which they live and in relation to their goals, expectations, standards, and concerns." ${ }^{\circ}$ Assessment should be done to relate the quality of life, disease burden and the effectiveness of treatment.

General health related QoL measure compare the effect of different conditions on patient's life. ${ }^{6}$ Dermatology specific questionnaires include DLQI, Skindex and Dermatology quality of life scales (DQLS). ${ }^{7}$ Finlay and Khan developed the widely used DLQI for use in research studies and routine clinical practice to assess the changes in HRQoL, as it is a sensitive measure. ${ }^{7}$ Acne specific measures include Acne Disability index (ADI), Cardiff Acne Disability Index (CADI), Assessment of the psychological and social effects of Acne (APSEA), and Acne Quality Of Life (AQOL). ${ }^{7}$ The CADI was developed to quickly assess the level of disability caused by acne. ${ }^{6}$ Clinical trials indicate that use of global and specific scales together has complementary benefits. ${ }^{8}$ Impact of acne in QoL of the patients, determines management of the disease.

The majority of studies on psychosocial impact of acne have been conducted among patient groups in US and Europe, but there is poor understanding of this among the Indian population. ${ }^{9}$ Increased awareness and early intervention for the psychosocial and psychiatric sequel of acne can benefit patients. ${ }^{10}$

\section{Materials and Methods}

This is a hospital based prospective cross-sectional questionnaire study done on acne vulgaris patients attending the dermatology outpatient department of Azeezia Medical College, Kollam, Kerala. Clinically diagnosed as Acne vulgaris of age group 16-35 were 
included, after obtaining their consent. Study Duration was 6 weeks (from 01/08/2017 to 15/09/2017).

Study tool: DLQI Questionnaire (Dermatology Life Quality Index Questionnaire): This is a standardized questionnaire prepared by Finlay and Khan in 2004, to study health related quality of life in various skin diseases.

CADI Questionnaire (Cardiff Acne Disability Index Questionnaire): This is also a standardized questionnaire prepared by Motley and Finlay in 1992, for assessment of quality of life in acne patients. It is designed for use in young adults and teenagers with acne.

There are different language versions for both the questionnaires, and can be used for specific populations.
Formal permission from the Department of Dermatology, Cardiff University was obtained to use the DLQI and CADI Questionnaires.

Data Collection: The purpose of the study was explained. Questionnaires were duly filled up by the patients after clinically diagnosing them as Acne vulgaris. It was made clear that the participation was voluntary. Collected questionnaires were analyzed.

Scoring: The DLQI is calculated by summing the score of each question resulting in a maximum of 30 and minimum of 0 . Higher the score, higher the quality of life is impaired.

\begin{tabular}{|l|l|}
\hline DLQI score & \multicolumn{1}{c|}{ Interpretation } \\
\hline $0-1$ & No effect at all on patient's life \\
\hline $2-5$ & Small effect on patient's life \\
\hline $6-10$ & Moderate effect on patient's life \\
\hline $11-20$ & Very large effect on patient's life \\
\hline $21-30$ & Extremely large effect on patient's life \\
\hline
\end{tabular}

The CADI is calculated by summing the score of each question resulting in a maximum of 15 and a minimum of 0 . Higher the score, greater the impairment in quality of life.

\begin{tabular}{|l|l|}
\hline CADI score & Interpretation (Degree of impairment) \\
\hline 0 & None \\
\hline $1-5$ & Mild impairment \\
\hline $6-10$ & Moderate impairment \\
\hline $11-15$ & Severe impairment \\
\hline
\end{tabular}

\section{Data analysis}

The DLQI and CADI scores were calculated. Data was entered in Microsoft Excel. Data analysis was done using the SPSS Software. CADI was the outcome variable and the independent variables were sociodemographic characteristics (age, gender and socio-economic status). DLQI and CADI scores were expressed as mean. For analysis age was categorized into 2 groups (16-19 and 20-35 years) and socio-economic status into 2 (APL and BPL). Pearson's chi-square coefficient was used as test of significance.

\section{Results}

The Quality of Life of 70 acne patients attending the outpatient department of Dermatology in a tertiary care center in South Kerala was studied. Age of the study population ranged from 16-35 years. Mean age was 25.5. The maximum number $(71.42 \%)$ belonged to the $20-35$ age group and the rest $(28.57 \%)$ belonged to the 16-19 age group. Majority of patients were females (67\%). Most of the acne patients who attended the OPD (62.85\%) belonged to the APL while the rest (37.14\%) belonged to BPL class.
Acne affected the quality of life in $82.9 \%$ of patients as assessed by the DLQI questionnaire. [Fig. $1 \&$ Table 1]. $17.1 \%$ of the acne patients had no effect on their quality of life. $30 \%$ of the patients suffered small effect, while $27.1 \%$ had moderate effect, $24.3 \%$ had very large effect, and only 1 patient (1.4\%) had a severe effect on quality of life. The mean DLQI score was 6.97.

Acne affected the quality of life in $94.3 \%$ of patients as assessed by the CADI questionnaire. [Fig. 2 \&Table 2]Based on this, $5.7 \%$ of acne patients had no impairment in quality of life, $45.7 \%$ patients had mild impairment, $40 \%$ patients had moderate impairment, and $8.6 \%$ had severe impairment of quality of life.

Significance was tested using Chi-square test. Age and CADI scores showed high significance $[\mathrm{p}<0.005]$. Gender and Socioeconomic status with CADI were not found to be significant. ( $p=0.214$ and $p=0.448$ respectively). Age was a highly significant factor in impairment of quality of life. Quality of life impairment was more among adolescent age group than among young adults. 


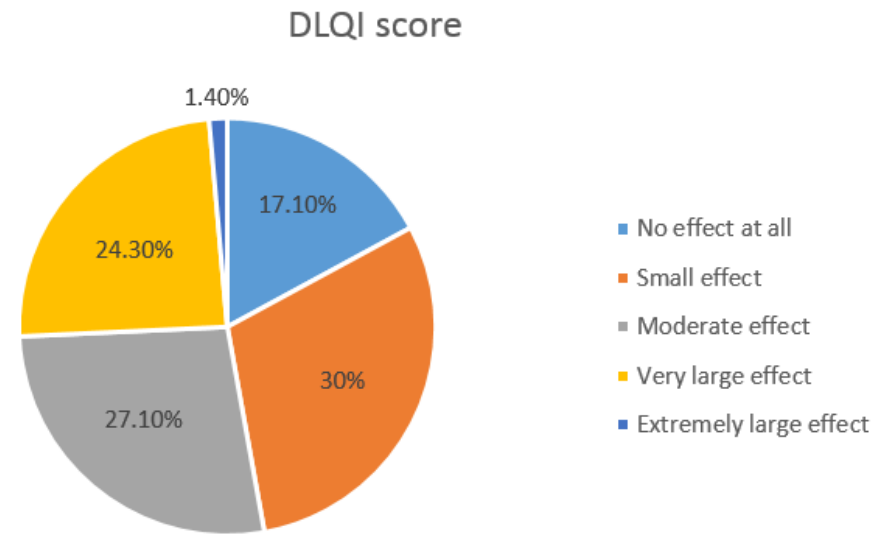

Fig. 1: Acne affected the quality of life in $\mathbf{8 2 . 9 \%}$ of patients as assessed by the DLQI questionnaire. (See table)

Table 1: showing DLQI scores based on gender. $17.1 \%$ of the acne patients had no effect on their quality of life. $30 \%$ of the patients suffered small effect, while $27.1 \%$ had moderate effect, $24.3 \%$ had very large effect, and only 1 patient $(1.4 \%)$ had a severe effect on quality of life.

\begin{tabular}{|l|c|c|c|c|c|c|}
\hline \multirow{2}{*}{\multicolumn{1}{|c|}{ DLQI }} & \multicolumn{2}{c|}{ Male } & \multicolumn{2}{c|}{ Female } & \multicolumn{2}{c|}{ Total } \\
\cline { 2 - 7 } & $\mathbf{N}$ & $\mathbf{\%}$ & $\mathbf{N}$ & $\mathbf{\%}$ & $\mathbf{N}$ & $\mathbf{\%}$ \\
\hline No effect (0-1) & 5 & 7.1 & 7 & 10 & 12 & 17.1 \\
\hline Small effect (2-5) & 6 & 8.5 & 15 & 21.4 & 21 & 30 \\
\hline Moderate effect (6-10) & 6 & 8.5 & 13 & 18.6 & 19 & 27.1 \\
\hline Very large effect (11-20) & 5 & 7.1 & 12 & 17.1 & 17 & 24.3 \\
\hline Extremely large effect (21-31) & 1 & 1.4 & 0 & 0 & 1 & 1.4 \\
\hline Total & 23 & 32.9 & 47 & 67.1 & 70 & 100 \\
\hline
\end{tabular}

\section{CADI Score}

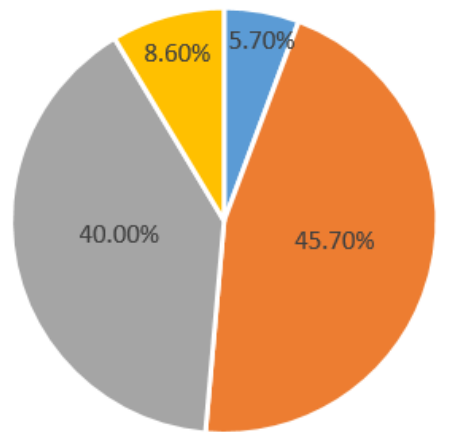

$$
\begin{aligned}
& \text { - No impairment } \\
& \text { - Mild impairment } \\
& \text { = Moderate impairment } \\
& \text { = Severe impairment }
\end{aligned}
$$

Fig. 2: Acne affected the quality of life in $94.3 \%$ of patients as assessed by the CADI questionnaire. (See table)

Table 2: showing CADI scores based on gender. Based on this, $5.7 \%$ of acne patients had no impairment in quality of life, $45.7 \%$ patients had mild impairment, $40 \%$ patients had moderate impairment, and $8.6 \%$ had severe impairment of quality of life

\begin{tabular}{|l|c|c|c|c|c|c|}
\hline \multirow{2}{*}{ CADI } & \multicolumn{2}{|c|}{ Male } & \multicolumn{2}{c|}{ Female } & \multicolumn{2}{c|}{ Total } \\
\cline { 2 - 7 } & $\mathbf{N}$ & $\mathbf{\%}$ & $\mathbf{N}$ & $\mathbf{\%}$ & $\mathbf{N}$ & $\mathbf{\%}$ \\
\hline None (0) & 1 & 1.4 & 3 & 4.3 & 4 & 5.7 \\
\hline Mild impairment (1-5) & 11 & 15.7 & 21 & 30 & 32 & 45.7 \\
\hline Moderate impairment (6-10) & 9 & 12.9 & 19 & 27.1 & 28 & 40 \\
\hline Severe impairment (11-15) & 2 & 2.9 & 4 & 5.7 & 6 & 8.6 \\
\hline Total & 23 & 32.9 & 47 & 67.1 & 70 & 100 \\
\hline
\end{tabular}


Table 3: showing independent variables and $p$ value

\begin{tabular}{|l|c|c|c|c|}
\hline \multicolumn{2}{|c|}{ Variables } & N & Df & p \\
\hline \multirow{2}{*}{ Age } & $16-19$ & 20 & \multirow{2}{*}{3} & \multirow{2}{*}{0.003} \\
\cline { 2 - 3 } & $20-35$ & 50 & & \\
\hline \multirow{2}{*}{ Gender } & Male & 23 & \multirow{2}{*}{3} & 0.214 \\
\cline { 2 - 3 } & Female & 47 & & \\
\hline \multirow{2}{*}{ SES } & APL & 44 & \multirow{2}{*}{3} & 0.448 \\
\cline { 2 - 3 } & BPL & 26 & & \\
\hline
\end{tabular}

\section{Discussion}

Acne can have serious psychosocial impairment and affect quality of life adversely. According to WHO, quality of life is defined as an individual perception of their position in the context of culture and value systems in which they live and in relation to their goals, expectations and concerns. Validated questionnaires are used for assessing the measurement of quality of life. Here, both dermatology specific questionnaire (Dermatology Life Quality Index) and acne specific questionnaire (Cardiff Acne Disability index) are used. DLQI, though a non-specific questionnaire is a validated questionnaire, and can be completed by patients without supervision. ${ }^{14}$ When assessed with DLQI questionnaire, 82.9\% and with CADI $94.3 \%$ showed impairment in quality of life. This is comparable to other studies. ${ }^{11-14}$ The mean DLQI score was 6.97, which is similar to other studies. ${ }^{6,11,12}$ In this study, the mean CADI is 5.8 which is comparable to the study conducted in Andhra Pradesh. ${ }^{6}$ On observation , there is moderate to severe impact on quality of life is $48.6 \%$ of patients based on CADI score and moderate to extremely large impact on the same for $52.8 \%$ patients assessed as DLQI score. In our study a female predominance was observed which could be attributed to the concern regarding their appearance when compared to other studies also. ${ }^{11}$ Certain other studies also showed male predominance. ${ }^{6}$

The males and females did not differ significantly in the mean age and the commonest age group was similar to other studies. ${ }^{9,11}$ Quality of life was more affected in adolescent age group, which was also found to be highly significant $(\mathrm{p}<<0.01)$. This result was similar to other studies. ${ }^{6,11,12,13}$ The mean age and commonest age group of both sexes didn't vary significantly and was similar to other studies. It was found that the quality of life is more affected in adolescent age group, and it was highly significant. This result was similar to other studies. Even though married young adults with children attribute to less impact in quality of life, it was not found to be statistically significant $(\mathrm{p}<0.05)$. Majority of affected patients belonged to upper class which focus more towards factors like life in cities, work and environment, making them more concerned on their appearance. This was not yet found to be statistically significant in our study ( $p>0.05$ ). Importance of assessing the quality of life has sufficient role for the dermatologist to define the treatment for the patient based on the severity in impairment of quality of life. There is relevance in providing proper counselling and psychosocial support to improve the quality of life in patients with acne, especially in adolescent age group.

\section{Conclusion}

Acne has definite implication in quality of life, the most affected category being the adolescent age group including school going and college fresher's who showed impairment in their quality of life. Acne has an outward influence in their social authorities, personal relationships and leisure activities which can result in unfavourable psychosocial disturbance. So, the identification of those patients with greater impact on quality of life so as to provide emotional support and appropriate counselling should be done by all practicing dermatologist.

\section{Limitations}

In the study, severity of acne is not assessed. Severity of acne and quality of life is directly proportional. As the duration of study was short, the sample size for assessing the quality of life in general was small, but adequate. This study cannot be generalized to a population even though most of the findings are comparable with other studies, because of small sample size and a hospital-based study. This was applicable for those who approach clinic, who definitely had a psychosocial impact.

\section{Recommendations}

DLQI and CADI can be used as a routine tool in Dermatology OPDs. Treatment and counselling can be based on the score obtained in these questionnaires and severity of acne.

Funding: No funding sources.

Conflict of interest: None declared.

\section{References}

1 Dawson AL, Dellavalle RP. Acne vulgaris. BMJ. 2013 May 8;346:f2634.

2 Kellett SC, Gawkrodger DJ. The psychological and emotional impact of acne and the effect of treatment with isotretinoin. Br J Dermatol 1999;140(2):273-82.

3 Sharma RK, Dogra S, Singh A, Kanwar AJ. Epidemiological patterns of acne vulgaris among adolescents in North India: A cross-sectional study and brief review of literature. Indian J Pediatr Dermatol 2017;18:196-201.

4 Kligman AM. Post adolescent Acne in Women. Cutis 1991;48(1):75-7. 
5 The World Health Organization Quality of Life Assessment (WHOQOL): Position Paper from the World Health Organization. SocSci Med 1995;41:1403-9.

6 Durai PC, Nair DG. Acne vulgaris and Quality of Life among Young Adults in South India. Indian J Dermatol 2015;60(1):33-40.

7 Barnes LE, Levender MM, Fleischer AB, Feldman SR. Quality of Life measures for Acne patients. Dermatol Clin 2012;30:293-300.

8 Jankovic S, Vukicevic J, Djordjevic S, Jankovic J, Marinkovic J. Quality of Life among school children with acne: Results of a cross-sectional study. Indian J Dermatol Venerol Leprol 2012;78:454-58.

9 Samanthula H, Kodali M. Acne and Quality of Life- A Study from a Tertiary Care Centre in South India. IOSRJDMS 2013;6:59-62.

10 Pandey P, Suresh MSM, Dubey V, Pandey P. A crosssectional study on Quality of Life among Acne vulgaris Patients. Int J Res Med Sci 2016;4:4800-5.
11 Swathylekshmy JL, Jayapalan S. Impact of Acne Vulgaris on the Quality of Life. J Evol Med Dent Sci 2017;618.

12 Hazarika N, Rajaprabha RK. Assessment of life quality index among Patients with Acne vulgaris in a Suburban Population. Indian J Dermatol 2016;61:163-8.

13 Yap FB. The Impact of Acne Vulgaris on the Quality of Life in Sarawak, Malaysia. 2012;16(2):57-60.

14 Finlay AY, Khan GK. Dermatology Life Quality Index (DLQI) - a Simple Practical Measure for Routine Clinical Use. Clin Exp Dermatol 1994;19(3):210-16.

How to cite this article: Pillai R., S. Sanoof, Mysa. A cross-sectional study on the assessment of dermatology life quality index among the adolescents and young adults with acne vulgaris in south Indian population. Indian J Clin Exp Dermatol 2018;4(4):314-18. 